\title{
Transnasal endoscopic Skull Base and brain surgery: surgical anatomy and its applications by Aldo C Stamm (2019) 690 pp., 704 illustrations hardback ISBN: 9781626237100 Thieme publishers New York/Stuttgart
}

\author{
P. di Russo ${ }^{1}$ - L. Giammattei ${ }^{1}$ - Sebastian Froelich ${ }^{1}$ \\ Received: 6 April 2020 / Accepted: 7 April 2020 /Published online: 28 April 2020 \\ (C) Springer-Verlag GmbH Austria, part of Springer Nature 2020
}

This book in its 2 nd edition is destined to become a milestone in the field of endoscopic endonasal surgery. Throughout its 665 pages and 72 chapters, the book represents a tour de force that covers all aspects of endonasal endoscopic surgery, from the original anatomy to the management of complications. On looking at the cover of the book, readers understand that the author aims to explore the entire skull base, giving special attention to anatomical details related to each endoscopic approach.

Generally, each chapter includes a brief introduction on the argument, a detailed focus on the specific topic and clear conclusions that help to fix the most important concepts. The presence of the Key-points Table focuses the attention of the reader and facilitates a rapid rereading of the chapter. Many anatomical images from different contributors accompany the text, embellishing several chapters. Moreover, twenty chapters come together with surgical videos, available online with the e-book, showing pertinent surgical cases.

The first two chapters of the book address the principles of transnasal endoscopic surgery. The anatomy of the nose, paranasal sinuses and skull base is elucidated by contributors from Rhoton's family. The chapters on preoperative assessment and postoperative care are helpful in order to review this aspect of surgery.

The subsequent parts of the book, from chapters III to XI, detail each single endoscopic endonasal approach from the anterior cranial fossa to the craniovertebral junction. The decision to start with transorbital approaches and to progress in the direction of the inferior clivus is logical and facilitates the

Sebastian Froelich

sebastien.froelich@aphp.fr

1 Department of Neurosurgery, Hôpital Lariboisière, Assistance Publique - Hôpitaux de Paris, Université de Paris, 2 rue Ambroise Paré, 75010 Paris, France comprehension of the reader. Also approved is the choice to treat other more complex approaches, as those directed to the infratemporal fossa, petrous apex and Meckel's cave and craniovertebral junction. The chapters are well constructed in an immediate and direct fashion. After the introduction, the specific indications and limitations of the approach are listed. The surgical technique is described in a step by step modality. Radiological and intraoperative pictures help to understand both the indications and the surgical anatomy of the approach. Finally, a brief conclusion summarises the concepts discussed in the chapter. It is pleasant to find in this book, focused on endoscopic surgery, a chapter discussing the controversial topic regarding the Endoscopic versus Microscopic approach for anterior skull base meningiomas. The chapter on Sinus and Skull Base Malignancies highlights with clear clinical examples the concept of cancer resection and the importance to have a multidisciplinary board.

The presence of a specific chapter on Paediatric Transnasal Endoscopic surgery also needs to be noted.

The last two chapters of the book cover the complex topic of complications related to conventional and expanded transnasal endoscopic approaches. The most commonly used reconstruction techniques for skull base defects are described. It was interesting to read the chapter on the management of internal carotid artery injury. There is also a specific section dedicated to post-operative endocrine complications.

Riding on an endoscope is a fascinating journey which starts inside the nasal cavity and, passing from the sella, the anterior and posterior cranial fossae to the craniovertebral junction and carries the reader into the deepest sides of the skull base.

This book will find passionate readers in both skilled neurosurgeons and ENT, including fellows and residents interested in improving their knowledge in endoscopic endonasal skull base. 